\title{
Erratum: Emergence of Correlated Optics in One-Dimensional Waveguides for Classical and Quantum Atomic Gases [Phys. Rev. Lett. 117, 143602 (2016)]
}

Janne Ruostekoski and Juha Javanainen

(Q) (Received 16 January 2018; published 26 January 2018)

DOI: 10.1103/PhysRevLett.120.049903

In the caption of Fig. 1 there is a misprint. The atom densities should be $\rho \lambda=2$ (dashed line) and $\rho \lambda=8$ (dotted line). The thickness of the sample $L=2 \lambda$. All other results and all conclusions remain unaffected. 